\title{
Evaluation of Tangible User Interfaces for Desktop AR
}

\author{
Andreas Dünser, Julian Looser, Raphaël Grasset, Hartmut Seichter, Mark Billinghurst \\ The HIT Lab NZ, University of Canterbury, Christchurch, New Zealand \\ \{andreas.duenser, julian.looser, raphael.grasset, hartmut.seichter, mark.billinghurst\}@hitlabnz.org
}

\begin{abstract}
In this work we evaluated the usability of tangible user interaction for traditional desktop augmented reality environments. More specifically, we compared physical sliders and tracked paddles, and traditional mouse input for a system control task. While task accuracy was the same for all interfaces, mouse input performed the fastest and input with a tracked paddle the slowest. Performance with the physical sliders fell between those two. We present these results along with various findings from user comments, and discuss how they may influence the design of future desktop AR systems.
\end{abstract}

Keywords- Augmented Reality; Tangible User Interface; Desktop AR; Tangible AR

\section{INTRODUCTION}

Augmented Reality (AR) has gained increasing public attention in recent years. This was facilitated by easy delivery systems (such as Flash web components) and promotional downloads, bringing AR to people's desktops. Whereas most traditional AR research has used head-mounted displays, this is simply not practical for home users who commonly have monitors and webcam peripherals. Therefore there is a need for researchers to explore suitable interaction techniques for 'Desktop AR' environments. This involves finding answers to what is the most appropriate interaction paradigm for this type of environment.

This paper presents an evaluation of different interaction techniques for a system control scenario which plays a key role in a wide range of applications. For example, in an authoring scenario, the user may need to adjust object size and material properties or in visualisation applications, scalar parameters may be adjusted for filtering data.

For this study we focused on Tangible Augmented Reality (TAR), an approach that combines the intuitive input of Tangible User Interfaces (TUIs) with the visualisation potential of AR [10]. TUIs create links between the physical environment and the virtual environment by binding digital information and meaning with everyday physical objects [1]. They support direct bimanual interaction [2, 3], eyes-free interaction, and provide passive haptic feedback [4, 5]. This allows users to focus on the task without being distracted by control mechanisms. AR interfaces generally provide intuitive and flexible ways of visualizing virtual content, but some systems offer little or no support for interacting with the content [6]. By merging TUIs with AR, TAR interfaces close the gap between interaction with computers and the physical world [7].

The TAR approach has often been used in desktop AR environments, typically by using tracked markers as the physical artefacts. However, few evaluations have been conducted to study their usability. Moreover, due to better accessibility to low level programming of hardware components, we have seen a burgeoning of physical components in the development of tangible interfaces. Nonetheless, they also have hardly been evaluated.

In this paper we conducted an evaluation to study the validity and usability of tangible interfaces in the context of a desktop AR system control task. Two interfaces with physical sliders were developed and empirically compared to more traditional input devices for desktop AR. After discussing the related work, we next present the experimental design and report the experimental findings.

\section{RELATED WORK}

Sliders are familiar components in many user interfaces, physical or virtual, $2 \mathrm{D}$ or $3 \mathrm{D}$. They allow the user to efficiently set a one-dimensional value and to clearly see where that value lies within the acceptable range.

Examples of hardware sliders include the onedimensional force feedback slider [8], and Chipman's SlideBar interface [9]. Ramos et al. [10] developed the Zliding interface for pressure-sensitive displays. Sliders are also commonly provided in the form of augmented (tracked) tools, for example by Asai et al. [11]. Using the occlusion of markers as an input modality, Lee et al [6] developed sliders composed of strips of small markers.

Comparing virtual and physical controls (buttons and rotation knobs) Fiebrink et al [12] found that physical controls were perceived as more accurate for continuous parameters than for discrete parameters, whereas this relationship was reversed for the virtual controls. Ware and Rose [13] found that creating conditions for natural eye-hand coordination can aid performance. Ideally the physical properties of an interaction device suggest how it should be used and constrain how the users can manipulate it [14].

In early studies of 3D input devices [15] Jacob and Sibert found that the proper design of an input device depends on how users perceive the attributes of a task. 3D tracked input devices worked best if all attributes were related or integrated, whereas for manipulating independent task attributes a mouse worked better. Better performance could be achieved if the task and device are both either integral or separable.

\section{INTERFACE EVALUATION}

The research literature demonstrates that some of the major factors for slider design are; physical feedback, continuous vs discrete tasks, hand-eye coordination and 
integral vs separated task. We are specifically interested in the impact of tactile feedback on user performance. In an AR interface you can spatially register a physical slider with a virtual one. Therefore we wanted to investigate effect of this visual registration on user performance and the benefits beyond a demonstrated intuitiveness for placement tasks [10].

Considering the work of Jacob and Siebert [15], a desktop AR based system control task was developed that includes separated selection and manipulation of virtual objects. Jacob and Siebert found that a separated interaction device such as a mouse works better for this kind of tasks rather than an integrated device such as a $3 \mathrm{D}$ tracked prop. Thus we assume that a mouse will outperform a tracked paddle, another commonly used interaction device for desktop AR systems. As an alternative to these interaction devices we developed two interfaces with physical sliders that offer different support in terms of integration or separation. Following the research literature, we hypothesize that the physical feedback afforded by these devices can improve user performance and satisfaction compared to mouse and tracked paddle input.

\section{A. Task, Interfaces and Apparatus}

A simple desktop AR application was built in which two $3 \mathrm{D}$ virtual models were displayed in front of the user. We used a monitor with a camera on top of the screen and mirrored video image on screen. The virtual objects were spatially positioned on the surface of a table (relative to standard ARToolKit [16] tracking markers). The user task was to change the scale and grayscale parameters of an input object (obj_input) to match that of a reference object (obj_target). To manipulate the parameters the user could control a set of virtual sliders displayed on the screen (see Figure 1).

Four competing techniques for controlling the virtual slider were developed (see Figure 2):

Mouse - this is the standard interface for desktop applications and commonly used for desktop AR. The 2D mouse position is projected into the $3 \mathrm{D}$ scene, allowing the user to interact with whichever object is under the cursor. In the evaluation interface, the user can click and drag on a slider to adjust its value.

Paddle - a cardboard paddle with a tracked marker as commonly used in AR interfaces for selection and interaction. The user could activate a virtual slider by moving the paddle within a threshold distance. While within the threshold distance, the value of the slider was bound to the location of the paddle. To disengage from the slider, the user moves the paddle away.

Slider - this is an extension to the paddle interface and consists of a tracked paddle with an integrated physical slider. A virtual slider is activated by the paddle's close proximity, but the value was changed by touching a physical sensor instead of moving the paddle. Similar to a mouse this is a separated device, so we expect it to outperform the paddle interface.

Two Sliders - this interface consists of two physical sliders, one for each parameter. The physical sliders were positioned to visually correspond with the virtual sliders. We assume that this interface allows users to perform the task rather quickly. This is because they do not have to move the interface to select the virtual sliders, and the interface allows bimanual input and eyes-free interaction.

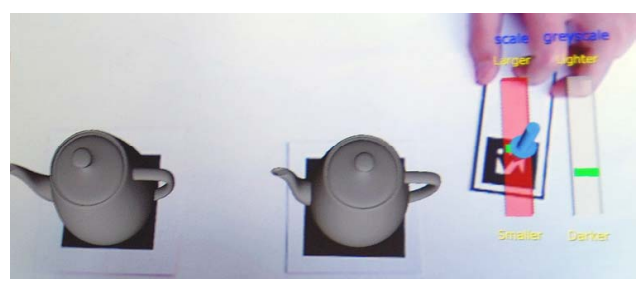

Figure 1. Scale and grayscale parameters of obj_input are adjusted with the virtual sliders.

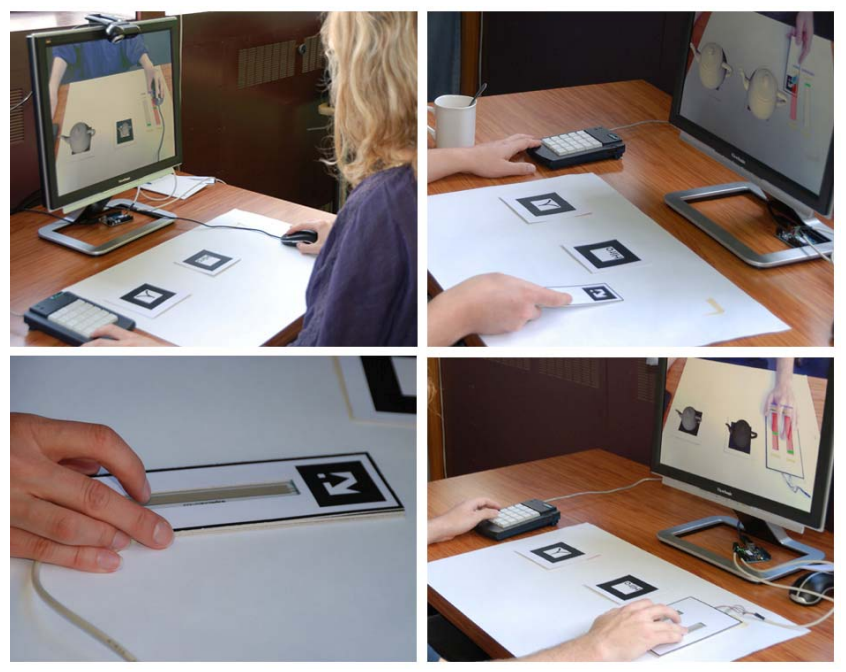

Figure 2. The four interfaces used in the experiment: Mouse, tracked paddle, tracked paddle with physical slider, and two sliders.

The sensors used were $10 \mathrm{~cm}$ long touch sensitive SoftPot sensor strips from Spectra Symbol ${ }^{1}$. The sensors output was monitored with an Arduino microcontroller board connected to a PC via USB. The SoftPot sensor provides an analog voltage reading that varies linearly corresponding to the point of pressure along its length. We chose an absolute mapping between this reading and the virtual slider value (each value of the virtual slider corresponds to a position on the physical slider).

The evaluation was conducted on a standard PC workstation running Windows XP Professional (Pentium 4 3.2GHz, 1GB RAM, 19" LCD at 1280x1024 pixels). For AR tracking, we used a Logitech Quickcam Pro 9000 camera, capturing at $640 \times 480$ pixels, 30 frames per second. The software was built using the osgART library [17], with underlying ARToolKit4 tracking.

We put a separate marker on the location where the target object was displayed to avoid perceptual differences between the target object and the input object. Without this additional marker we found that visual differences in background

\footnotetext{
${ }^{1}$ http://spectrasymbol.com/typo3/site/en/softpotsplash/softpot.html
} 
contrast made it difficult to compare the grayscale between the two objects.

Participants used all four interfaces to complete two practice and twelve measured trials. Interfaces were presented according to a balanced Latin-square. Each trial began with the input object in a default state where both slider values were at zero, and the target object set to a random scale and grayscale combination. For all interfaces the possible range of virtual slider values was limited to 32 levels for both scale and grayscale. After adjusting the sliders so that input and target objects matched, the participant then pressed a key on a keypad to indicate they were finished (see Figure 2). Participants were instructed to work as quickly and accurately as possible. Accuracy for both parameters was measured based on the absolute error between their chosen values and the target values. Task completion time was measured from the start of the trial until the last validation for the last slider adjustment.

After completing all trials the participants filled out a questionnaire with questions on ease of use and overall interface ranking, and space for general comments. Users ranked their agreement with three statements: "The interface was easy to use", "It was easy to operate the virtual sliders", "The interface was appropriate for the task".

Sixteen post graduate university students (computer science, interaction design, engineering, psychology) participated in the experiment (eleven males and five females) ranging from 21 to 51 years with a mean age of 29.5 years. The participants' previous AR experience ranged from none to over 500 hours.

\section{B. Results}

The effect of interface type on scale accuracy, grayscale accuracy and task completion time were analyzed with repeated measures ANOVA for each of the dependent variables.

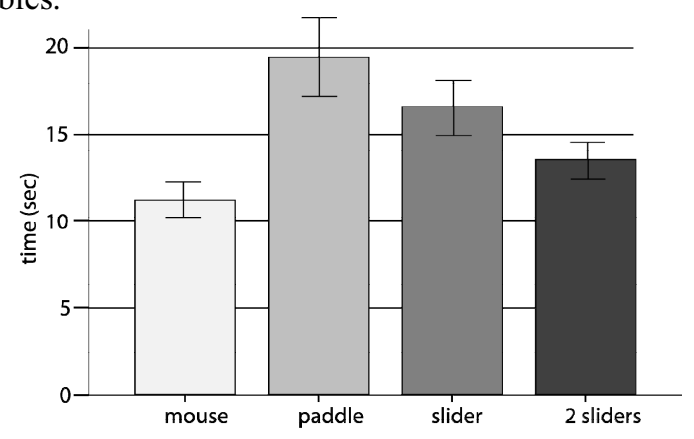

Figure 3. Task completion times $+/$ - SE

We found no significant effect of interface on either scale accuracy or grayscale accuracy. Both were almost the same for all interfaces with scale accuracy ranging from $96.76 \%$ (paddle) to $97.27 \%$ (slider) and grey scale accuracy from $94.12 \%$ (paddle) to $94.60 \%$ ( 2 sliders). However, we found a significant effect of interface on task completion time $\left(F_{2.26,33.96}=18.59, p<.01\right)$ (see Figure 3). Post hoc multiple comparisons (with Bonferroni adjustment) showed that mouse interaction $(M=11.20$ seconds, $S D=4.21)$ was significantly faster than all other interfaces. The paddle showed the longest task completion time ( $M=19.44 \mathrm{sec}$, $S D=9.16)$ and was significantly different from all but the slider interface $(M=16.57 \mathrm{sec}, S D=6.40)$. The two sliders interface $(M=13.51$ seconds, $S D=4.32)$ was significantly faster than the paddle, but slower than the mouse and not different from the single slider.

Significant differences between the interfaces were found for all questionnaire questions (see Figure 4) ("The interface was easy to use" $\left(\chi^{2}=29.89, d f=3, p<.01\right)$; "It was easy to operate the virtual sliders" $\chi^{2}=35.30, d f=3, p<.01$; "The interface was appropriate for the task" $\chi 2=28.71, d f=3$, $p<.01)$. A post hoc analysis for the two questions on ease of use and ease of virtual slider operation showed similar results, with the mouse and two slider interfaces being rated significantly higher than the paddle and slider interfaces. With respect to appropriateness for the task, the two sliders interface was rated higher than the paddle and the slider, and the mouse differed only from the paddle.

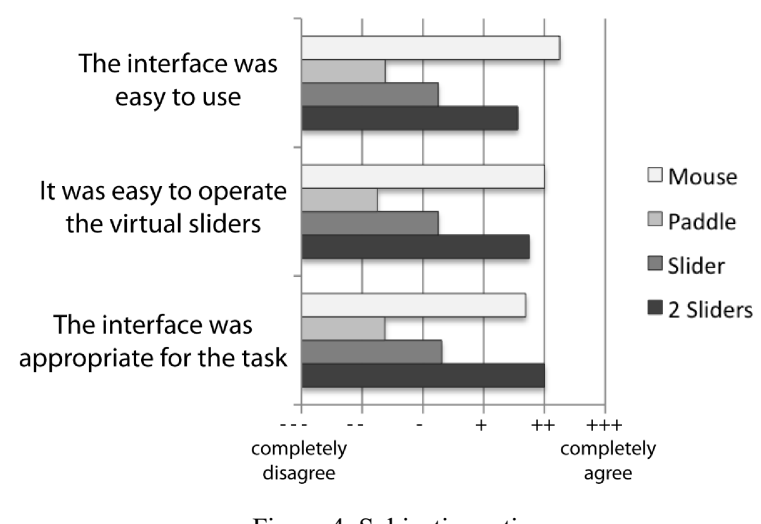

Figure 4. Subjective ratings

Overall the users preferred the mouse and two sliders interface over the other two interfaces $\left(\chi^{2}=29.89, d f=3\right.$, $p<.01)$.

\section{Observations and user comments}

Desktop AR environment: As expected, the main problem with desktop AR setup was the perception of forwards and backwards movement. This was confusing for most participants and more problematic for interfaces that involved movement to operate the sliders (mouse, paddle). However, it becomes less of an issue once the users are familiar with the mapping and no longer need to look at the physical interface while manipulating it. Not having to look at the interface during manipulation was one advantage of the sliders which some participants commented on.

The paddle interface: Most problems were reported for the paddle interface. Due to some jitter in the tracking the pointer sometimes twitched, slightly changing the parameter value. Interacting with the paddle was problematic because there was no mechanism to 'lock' a value for a parameter. To overcome this issue some experienced AR users covered the marker with a finger after changing the parameter to the desired value, thereby 'locking' it in. Changing between the parameters was another problem for some users, who would 
exit from one virtual slider and accidentally enter the selection area of the other slider, modifying its value.

Slider Interfaces: One difficulty with the physical sliders was to judge the exact pressure needed to operate them. Too little pressure could cause the virtual slider to 'jump', giving a feeling of crude sensitivity. Some participants chose to tap on different positions on the sliders to change values instead of using continuous sliding movements. Most participants felt that the absolute mapping between physical and virtual slider was unintuitive. One participant commented that "you control the paddle like a mouse", so he would expect the slider to behave like a mouse wheel. Only few participants used both sliders of the two sliders interface simultaneously. The one slider interface was used either with one hand (thumb and middle or ring finger holding the paddle and index finger operating the slider, see Figure 2) or two hands, with the non-dominant hand holding the paddle in place.

\section{DISCUSSION AND CONCLUSION}

Our experiment aimed at studying how standard user interfaces and new kinds of tangible user interfaces compare in a desktop AR based system control task. We found no difference in user accuracy between the input interfaces; however task completion times did differ. We instructed the participants to work as quickly and accurately as possible, so this result may indicate that they put more effort into being accurate rather than being fast. However, they could achieve the same level of accuracy more quickly with the mouse than with the other interfaces. A computer mouse is a standard user interface. Users are well acquainted with it and most likely use this device on a daily basis. The other interaction devices are new and therefore users might not be able to interact as efficiently. However, as we also uncovered, mice have certain shortcomings when used in desktop AR environments. Ultimately our task only required movements in two dimensions and was therefore rather similar to regular desktop interaction. For these task types mice perform well. On the other hand the tracked paddle, seems less appropriate for these tasks but is more suitable as a $3 \mathrm{D}$ input device.

Similar to Jacob and Siebert [15] we also found that matching of interface and task allows for improved performance. A mouse (separated input device) worked best for the separated task. This did not hold true for the slider interface as it was not faster than the paddle. However, to some extent this can be explained by the interface usability issues we found.

The two-slider interface performed not quite as well as the mouse but better than the paddle input. It was slightly, although not significantly, faster than the one slider interface. Having one dedicated slider for each parameter made the interface affordances immediately obvious and meant the users did not have to change anything to select parameters, which saved time and effort. While this result is expected, the one slider interface is more flexible and offers better scalability as the number of parameters increases.
We found that the absolute slider mapping was not very intuitive, especially for the interface with one slider. This could be because people are used to the relative mapping of mouse scroll wheels and also because of the type of physical sliders we used (touch-sensitive, with no absolute physical slider position). In future studies we will include a relative slider mapping to explore if we can create a more intuitive interface. Furthermore, we plan to compare these interfaces to other approaches we are developing using computer vision based approaches for sliders.

\section{REFERENCES}

[1] H. Ishii and B. Ullmer, "Tangible bits: towards seamless interfaces between people, bits and atoms," Proc. CHI'97 Atlanta, Georgia, United States: ACM, 1997.

[2] G. W. Fitzmaurice, H. Ishii, and W. A. S. Buxton, "Bricks: laying the foundations for graspable user interfaces," Proc. CHI'95 Denver, Colorado, United States: ACM Press, 1995.

[3] M. Crider, S. Bergner, T. N. Smyth, T. Muller, M. K. Tory, A. E. Kirkpatrick, and D. Weiskopf, "A mixing board interface for graphics and visualization applications," Proc. GI '07 Montreal, Canada: ACM, 2007.

[4] S. J. Henderson and S. Feiner, "Opportunistic controls: leveraging natural affordances as tangible user interfaces for augmented reality," Proc. of the VRST'08 Bordeaux, France: ACM, 2008.

[5] B. Ullmer, H. Ishii, and R. J. K. Jacob, "Token+constraint systems for tangible interaction with digital information," ACM Trans. Comput.Hum. Interact., vol. 12, pp. 81-118, 2005.

[6] G. Lee, M. Billinghurst, and G. J. Kim, "Occlusion based interaction methods for tangible augmented reality environments," Proc. VRCAI '04 Singapore: ACM, 2004, pp. 419-426.

[7] H. Kato, M. Billinghurst, I. Poupyrev, I. Tetsutani, and K. Tachibana, "Tangible Augmented Reality for Human Computer Interaction," Proc. Nicograph Nagoya, Japan, 2001

[8] A. Shahrokni, J. Jenaro, T. Gustafsson, A. Vinnberg, J. Sandsjo, and M. Fjeld, "One-dimensional force feedback slider: going from an analogue to a digital platform," Proc. NordiCHI'06, Norway: ACM, 2006.

[9] L. E. Chipman, B. B. Bederson, and J. A. Golbeck, "SlideBar: analysis of a linear input device," Behav. Inf. Technol., vol. 23, pp. 1-9, 2004.

[10]G. Ramos and R. Balakrishnan, "Zliding: fluid zooming and sliding for high precision parameter manipulation," Proc. UIST'05 Seattle, WA, USA: ACM, 2005.

[11]K. Asai, T. Kondo, H. Kobayashi, and A. Mizuki, "A Geographic Surface Browsing Tool Using Map-based Augmented Reality," Proc. Visualization Conference USA: IEEE, 2008, pp. 93-98.

[12]R. Fiebrink, D. Morris, and M. R. Morris, "Dynamic mapping of physical controls for tabletop groupware," Proc. CHI'09 Boston, MA, USA: ACM, 2009.

[13]C. Ware and J. Rose, "Rotating virtual objects with real handles," ACM Trans. Comput.-Hum. Interact., vol. 6, pp. 162-180, 1999.

[14] K. Hinckley, R. Pausch, J. C. Goble, and N. F. Kassell, "A survey of design issues in spatial input," Proc. UIST'94 Marina del Rey, California, United States: ACM, 1994.

[15]R. J. K. Jacob and L. E. Sibert, "The perceptual structure of multidimensional input device selection," Proc. CHI'92 Monterey, California, United States: ACM, 1992.

[16]H. Kato and M. Billinghurst, "Marker tracking and hmd calibration for a video-based augmented reality conferencing system," Proc. IWAR99, 1999, pp. 85-94.

[17]J. Looser, R. Grasset, H. Seichter, and M. Billinghurst, "OSGART - A Pragmatic Approach to MR," Proc. ISMAR '06, Santa Barbara, CA, USA, 2006 